\title{
CYLINDER HEAD FEM ANALYSIS AND ITS IMPROVEMENT
}

\author{
Shixiong $\mathrm{Li}^{1, *}$, Jinlong Mao ${ }^{1}$, Shumao Wang ${ }^{1}$ \\ ${ }^{1}$ College of Engineering, China Agricultural University, Beijing, China, 100083 \\ * Corresponding author, Address: P.O. Box 114, College of Engineering, China Agricultural \\ University, 17 Tsinghua East Road, Beijing, 100083, P. R. China, Tel: +86-10-62736460, \\ Fax:+86-10-62736385,Email: li_shixiong@263.net, lsx@cau.edu.cn
}

Abstract: The nose bridge between the inlet valve and exhaust valve is the most fragile part on the cylinder head. Fatigue crack is the major failure mode. It is necessary to analyze the structure strength and reliability (SSR) of this zone. In this paper, a three-dimensional (3D) model for a engine cylinder head is built up using Pro/Engineer wildfire 2.0, and the 3D FEM analysis of the SSR is carried out. The temperature field and stress field of the nose bridge are also calculated based on the model. Several improvement designs are given and compared as the result of the above analysis, which lays the foundation for the cylinder head production.

Keywords: engine, cylinder head, nose bridge zone, SSR, FEM, design improvement

\section{INTRODUCTION}

Computer technology has been widely used in the agricultural area, especially computer simulations, which greatly promote engineering analysis. With the improvement of the computer's capability, the finite element method (FEM) is considered to be one of the most powerful design tools in Computer-Aided Engineering (CAE) (Liu, 2004). Using this method, the complex structural configuration can be modeled and the response at any point of the structure can be easily determined (Cyuan, 2000). Now, the finite element method has been evolving to be a widely accepted tool for the solution of pragmatic engineering problems (Liang, 2004). 
The cylinder head is one of the most complex parts in the engine, and it endures high thermal and mechanical load during working cycles (Zhang, 2002). The nose bridge on the head between the inlet valve and exhaust valve is subjected to the hot air through the exhaust valve and the cool air through the inlet valve simultaneously, and it is the weakest part in the cylinder head. The fatigue in the nose zone is one of the most important factors that shorten the life of a cylinder head. So it is very meaningful to find a method to weaken restriction of the heat distortion in the nose zone, especially in the preliminary design stage, the using of computer simulation to analyze the strength and fatigue life of a cylinder head is very important and necessary. In this paper, a successful and feasible numerical model was configured first and analyzed thoroughly, and then with the result got from the analysis, several ameliorative models are analyzed based on finite element method.

\section{MODELLING AND COMPARISION ANALYSIS}

Due to the complexity of cylinder head in geometrical structure and the heat load, the Pro/Engineer Wildfire 2.0 is selected as the three-dimension solid modeling tool. One cylinder area on the cylinder head of diesel engine 4105Q was modeled with it and the model was transferred to the threedimensional finite element analysis software ANSYS 9.0 to do the further analysis.

\subsection{The model of the cylinder head}

The three-dimensional model created by Pro/Engineer Wildfire 2.0 (Fig. 1) made some simplify for some structures which have no large effect on the temperature and stress field distribution, such as bolt holes, pin holes and ribs etc. But there are no simplify on the major structure dimensions and shapes in order to have an accuracy correct analysis result.

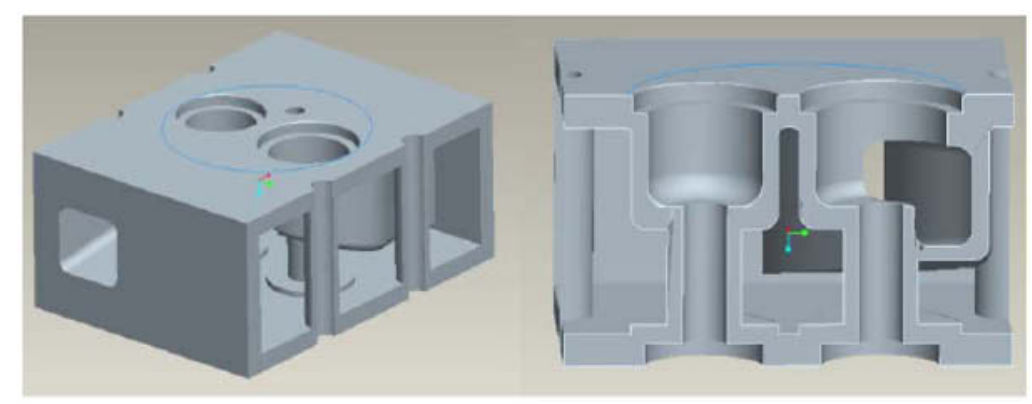

Fig. 1. 3D cylinder head model 
When the model is completed, save it to the file of igs and transfer it to the finite element analysis (FEA) software ANSYS 9.0. In the FEA software, after defining the properties of the material and the elements, the model is meshed by using automatic mesh tool. Fig. 2 show the meshed model, which contains 47310 elements and 84498 nodes.

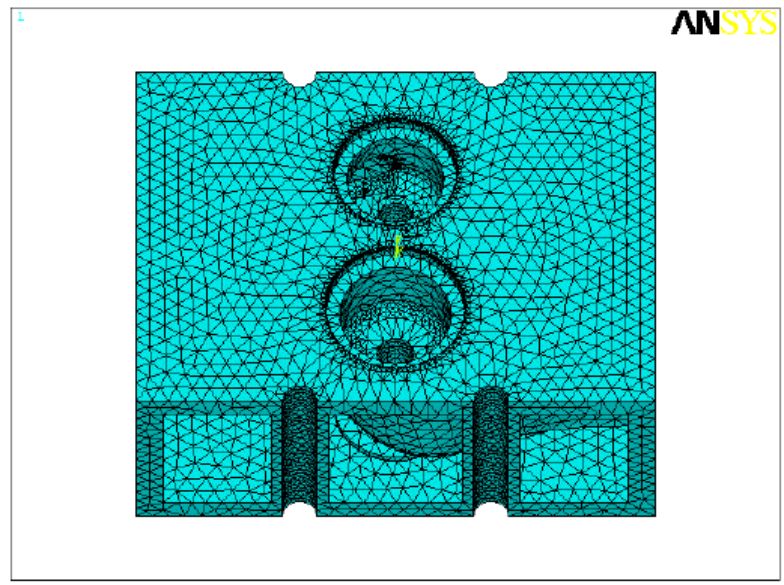

Fig. 2. The meshed model of the cylinder head

\subsection{The comparison analysis of the original and the improved designs}

The main objective of this study is to provide information on the structure and thermal stress of the cylinder head nose bridge and provide feasible solutions on the structure design improvement.

\subsubsection{The temperature and stress field calculation of the original design}

In order to calculate the temperature and stress distributions in the cylinder head, the third-type boundary condition should be defined first and then as the thermal load applied on the FEA model.

The third-type boundary condition:

$$
k_{x} \frac{\partial T}{\partial x} n_{x}+k_{y} \frac{\partial T}{\partial y} n_{y}+k_{z} \frac{\partial T}{\partial x} n_{z}=\alpha\left(\mathrm{T}_{\infty}-\mathrm{T}\right)
$$

where:

$\alpha$ : convection coefficient;

$\mathrm{k}$ : heat conduction coefficient;

To: the liquid temperature;

$\mathrm{T}$ : the temperature on the part surface;

nx, ny, nz: the direction cosine of the normal to the boundary. 
The boundaries are defined through many modification and calculation using the established experiential equation and values shown in Table 1.

Table 1. The Experiential Values of $\alpha$ and $\mathrm{T}$

\begin{tabular}{lcc}
\hline Location & $\alpha-W /\left(m^{2} \cdot K\right)$ & $\mathrm{T}$ \\
\hline Upper and side surface of the cylinder head & 23 & $293 \mathrm{~K}$ \\
The surface of air intake channel & 350 & $335 \mathrm{~K}$ \\
The surface of exhaust gas channel & 650 & $973 \mathrm{~K}$ \\
The surface of combustion chamber & 1000 & $1200 \mathrm{~K}$ \\
The surface of coolant channel & 3000 & $353 \mathrm{~K}$ \\
Immediately below the nose bridge & 3600 & $383 \mathrm{~K}$ \\
Forced cooling & 6000 & $383 \mathrm{~K}$ \\
Cylinder head with the contact surface of the inlet valve seat & 150 & $665 \mathrm{~K}$ \\
Cylinder head with the contact surface of the exhaust valve seat & 200 & $803 \mathrm{~K}$ \\
Cylinder head, gasket and cylinder block & 100 & $503 \mathrm{~K}$ \\
\hline
\end{tabular}

The calculation results are shown in Fig. 3.

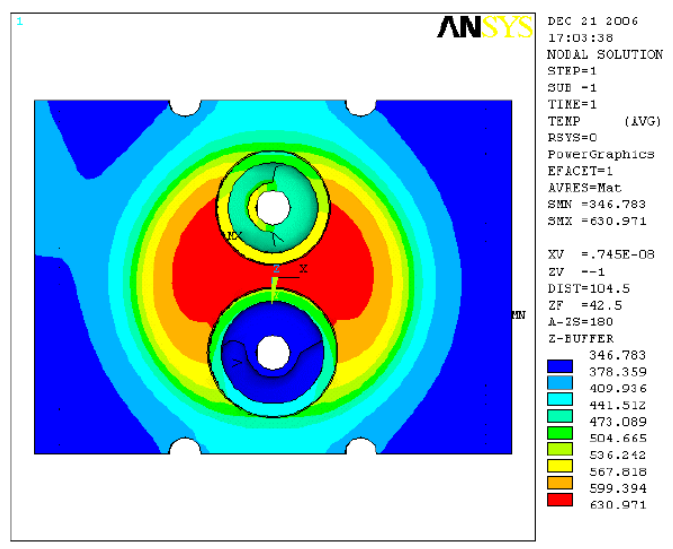

Fig. 3. Temperature distribution of the original design

In order to examine the plausibility of the boundary selection, the calculation results of the temperature distribution and the experimental results of Type Z6110 diesel engine are compared, as shown in Table 2.

Table 2. The Comparison between Calculation and Experimental Temperatures

\begin{tabular}{lccccccccc}
\hline Points & 1 & 2 & 3 & 4 & 5 & 6 & 7 & 8 & 9 \\
\hline Measurement & 483 & 424 & 518 & 507 & 558 & 605 & 613 & 471 & 430 \\
Calculation & 496 & 430 & 567 & 552 & 595 & 620 & 631 & 536 & 433 \\
Errors (\%) & 2.7 & 1.4 & 9.5 & 8.9 & 6.6 & 2.5 & 2.8 & 13.8 & 0.7 \\
\hline
\end{tabular}

The units for Temperatures are $\mathrm{K}$. 
Table 2 show the overall temperature distribution is coherent, which means the boundary condition selection is reasonable.

The thermal stress distribution is achieved through automatic data coupling calculation based on the temperature distribution calculation results. The calculation results of the thermal stress distribution are shown in Fig. 4.

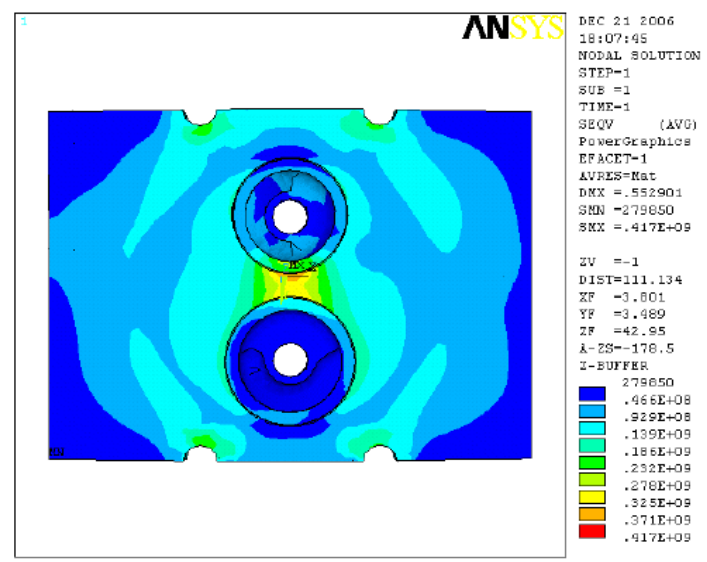

Fig. 4. Thermal stress distribution of the original design

Fig. $3 \& 4$ show that the max data occurs on the exhaust valve side of the nose bridge which need to find ways to lower its temperature and thermal stress.

\subsubsection{The temperature and stress field calculation of the improved designs}

In order to lower the temperature and thermal stress of the nose bridge zone, decrease the restriction of heat distortion, several models with different structure are provided.

Case1. Reduce the thickness of the nose bridge to $8 \mathrm{~mm}$;

Case2. Machining a deep and narrow arc groove (unload groove) with about $0.5 \mathrm{~mm}$ width in the nose bridge;

Case3. Machining a $3 \mathrm{~mm}$ diameter cooling water hole to improve the cooling condition in the nose bridge;

Case4. Casting a coolant channel whose outlet is opposite to the nose bridge.

The temperature distribution calculation results are shown in Fig. 5 


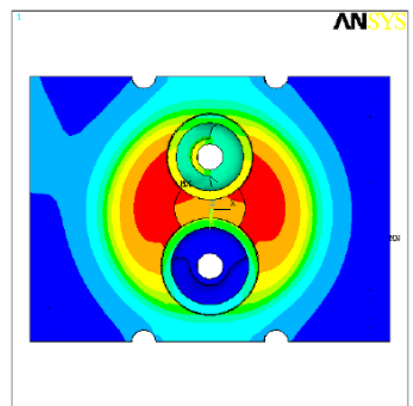

(a) Case1

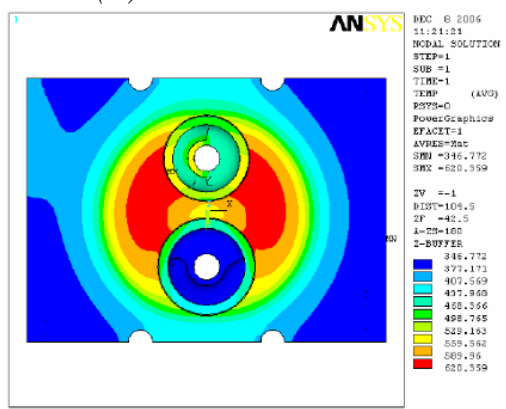

(c) Case3

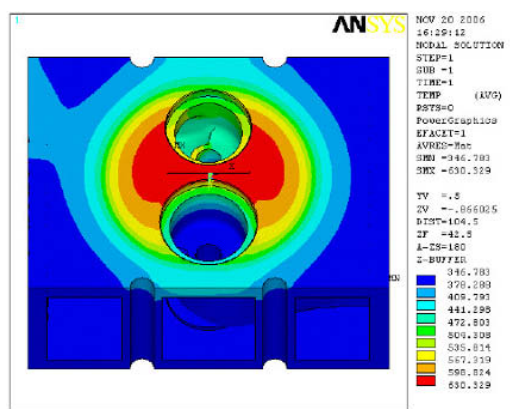

(b) Case2

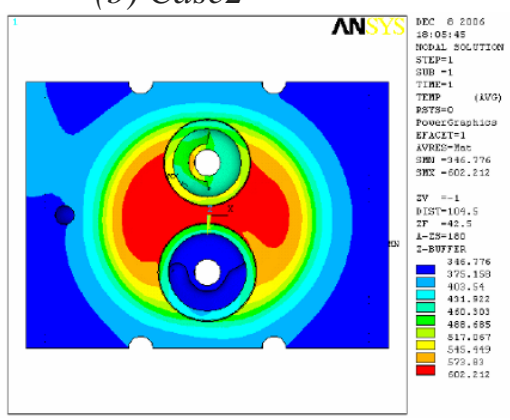

(d) Case 4

Fig. 5. The temperature distribution

The thermal stress distribution calculation results are shown in Fig. 6.

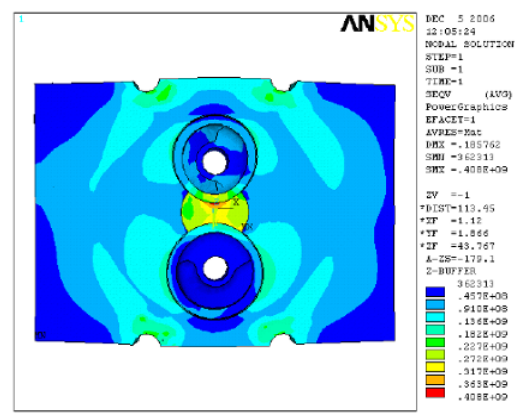

(a) Case 1

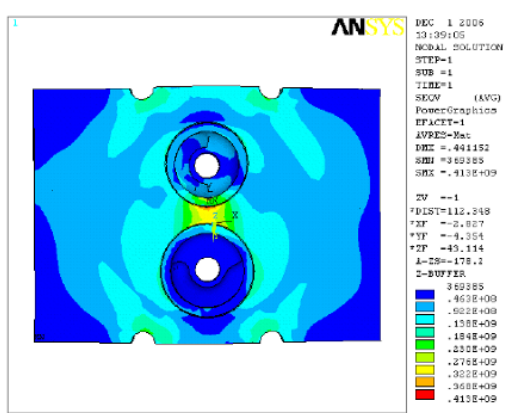

(c) Case 3

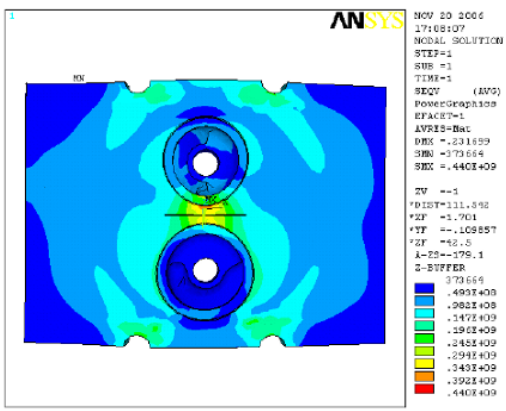

(b) Case2

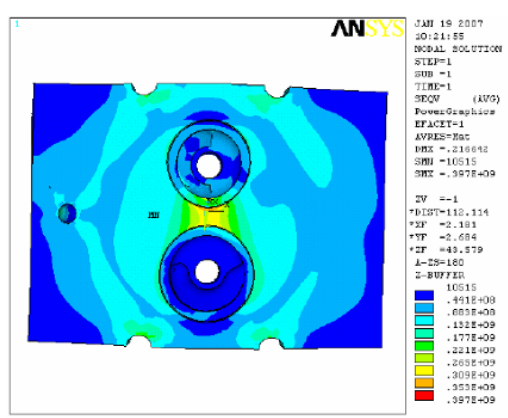

(d) Case4

Fig. 6. The thermal stress distribution 


\subsection{Comparison}

The comparison of the four improved design solutions' temperature and thermal stress distribution calculation results are shown in table 3 and table 4. (Note: $\mathrm{A}$ is the original case)

Table 3. The Highest Temperature Distribution Comparisons in Nose Bridge Zone

\begin{tabular}{lccccc}
\hline Cases & A & 1 & 2 & 3 & 4 \\
\hline The Highest Temperature $(\mathrm{K})$ & 630 & 610.4 & 630 & 590 & 602 \\
\hline
\end{tabular}

Table 4. The Highest Von Mises Thermal Stress Distribution Comparisons in Nose Bridge Zone

\begin{tabular}{lccccc}
\hline Cases & A & 1 & 2 & 3 & 4 \\
\hline The Highest Thermal Stress (MPa) & 417 & 408 & 440 & 413 & 397 \\
\hline
\end{tabular}

\section{RESULTS AND DISCUSSION}

Case1. Reducing the thickness of nose bridge improves the heat exchange condition in the nose zone, lowering the nose temperature about $20 \mathrm{~K}$ and the thermal stress about $9 \mathrm{Mpa}$.

Case2. Machining a unload groove have no change to the temperature distribution, while make the thermal stress increased about 23Mpa.

Case3. Machining a coolant holes in the nose bridge effectively lower the nose temperature $40 \mathrm{~K}$, and at the same time lower the thermal stress about 4Mpa.

Case4. Connect a coolant injector against the nose bridge is the best way to improve the temperature and thermal stress distributions in these solutions. It not only makes the temperature lower $28 \mathrm{~K}$, but also makes the thermal stress lower 20Mpa. It has been already found its application on some engines.

\section{CONCLUSIONS AND FUTURE WORKS}

From the above analysis, the main outcomes can be outlined as follows:

(1) For the nose bridge of cylinder head, an elaborate temperature field and thermal stress analysis was carried out using ANSYS 9.0. Results from the research could be provided to the designers to be as the reference in their work of improving the fatigue life of the cylinder head. 
(2) Just as what have been calculated, in all the cases the nose bridge between the inlet valve and exhaust valve are found to endure the highest temperature and highest thermal load. And the thermal load is dynamic and has a very high rate of repetition, causing the nose bridge to be the most fragile part in the cylinder head. We can know from the results that remove some metal and cool this zone with coolant will effectively lower the temperature and thermal stress.

(3) Because the configuration of cylinder head is too complex to predict the critical area and failure mode in the design phase, the finite element simulation have become the best method to obtain the stress distribution with different structures, this can greatly reduce project span time and total project cost.

\section{ACKNOWLEDGEMENTS}

This study is supported by China National 11th 5-Year Planned Project 'The Research on Rice and Wheat Cross-area Harvest Mechanization Technology' (Contract Number: 2006BAD28B03).

\section{REFERENCES}

Liu Jinxiang, LiaoRi dong, Zhang You. Finite Element Analysis for Cylinder Head of 6114 Diesel Engine. Transaction of CSICE, 2004, 22 (4): 367-372

Shiang-Woei Cyuan. Finite element simulation of a twin-cam 16-valve cylinder structure. Finite elements in Analysis and Design, 2000, (35): 199-212

S.L. Liang, X.H. Dai, H.M. Yao. 3D FE Analysis of Cylinder Head for Diesel Engine. Agricultural Mechanical Paper, 2004, 35 (3): 45-48

Zhang Weizheng, Zhang Guohua, Guo Liangping. The Thermal Fatigue Test and Modification of High-Temperature Creep for cast Iron Cylinder Heads. Transaction of CSICE, 2002, 23 (6): 67-69 